\title{
Effects of Mass Transfer and Heat Flux on Convective Flow Past a Moving Vertical Cylinder with Chemical Reaction
}

\author{
R. K. Deka and Arun Chaliha
}

\begin{abstract}
This paper presents an exact solution to onedimensional unsteady natural convection flow over a moving vertical cylinder under the combined buoyancy effects of heat flux and mass transfer along with chemical reaction is investigated. The dimensionless governing equations are solved by Laplace Transform technique. Graphical results for the velocity profiles, temperature, concentration, skin friction and Sherwood number are obtained and discussed for various physical parameters such as the chemical reaction parameter, Prandtl number, Schmidt number, thermal Grashof number, mass Grashof number and time
\end{abstract}

Index Terms-Natural convection, mass transfer, heat flux, chemical reaction, vertical cylinder and Laplace transform.

\section{INTRODUCTION}

In most chemical reactions, the reaction rate depends on the concentration of the species itself. In many chemical engineering processes there is a chemical reaction between a foreign mass and a fluid. These processes take place in numerous industrial applications such as manufacturing of ceramics, food processing and polymer production. The effects of mass transfer on flow past an impulsively started infinite vertical plate under constant heat flux condition along with chemical reactions were studied by Das et al. [1]. The dimensionless governing equations were solved by the usual Laplace Transform method. Ganesan and Loganathan [2] studied free convection boundary layer flow of a viscous and incompressible fluid past an impulsively started semi-infinite vertical cylinder with uniform heat and mass fluxes and chemically reactive species. Numerical solutions were derived by the finite-difference scheme of Crank-Nicolson type. On diffusion of chemically reactive species in convective flow along a vertical cylinder has been investigated by Ganesan and Rani [3].The authors discuss the Nusselt number, Sherwood number for both generative and destructive reaction. Muthucumaraswamy and Shankar [4] studied the first order chemical reaction on unsteady flow past a uniformly accelerated isothermal infinite vertical plate with heat and mass transfer in the presence of thermal radiation. The interaction of free convection with thermal radiation of a viscous incompressible unsteady flow past a moving vertical cylinder with heat and mass transfer is analyzed by Ganesan and Loganathan [5] . Transient free

Manuscript received April 15, 2012; revised May 27, 2012.

R. K. Deka is with the Department of Mathematics, Gauhati University, Guwahati-14, Assam

A. Chaliha is with the Department of Mathematics, Biswanath College, Sonitpur, Assam (e-mail: arunchaliha@rediffmail.com) convection about vertical plates and circular cylinders was studied by Goldstein and Briggs [6]. They discussed the transient free convection, heat transfer problem from vertical flat plate and vertical circular cylinders to a surrounding initially quiescent fluid .Also the velocity field and penetration distance in integral form were given for a vertical circular cylinder surrounded by a fluid of arbitrary Prandtl number.

In this study, we assume that a chemically reactive species is emitted from the surface of the vertical cylinder and diffuses into the fluid. It is also assumed that reaction takes place entirely in the stream. Due to the importance in many applications in the field of science and technology, the present study is required. It is proposed to study convective flow moving in a vertical cylinder under the combined effects of heat flux and mass transfer along with first order chemical reaction. The solutions are obtained by Laplace Transform technique for the velocity, temperature and concentration fields and these are presented in graphs. Skin friction and Sherwood number are also obtained and illustrated graphically

\section{MATHEMATICAL ANALYSIS}

Consider unsteady laminar and viscous flow past a moving semi-infinite vertical cylinder of radius $r_{0}$ with uniform heat flux and mass diffusion. Here the $\mathrm{x}$-axis is taken along the axis of the cylinder in the vertically upward direction, and the radial coordinate $r$ is normal to it. Initially, it is assumed that the cylinder and fluid are at same temperature $T_{\infty}^{\prime}$ and concentration $C_{\infty}^{\prime}$.At $t^{\prime}>0$, the cylinder starts to move in the vertical direction with constant velocity $\mathrm{u}_{0}$. The concentration level near the cylinder is raised to $C_{w}^{\prime}$.It is also assumed that there exists a homogeneous first order chemical reaction between the fluid and species concentration. Here we assume the level of species concentration to be very low and hence heat generated during chemical reaction can be neglected. With these assumption the governing boundary layer equations can be written as

$$
\begin{gathered}
\frac{\partial^{2} u}{\partial r^{2}}+\frac{1}{r} \frac{\partial u}{\partial r}+\frac{g \beta\left(T^{\prime}-T_{\infty}^{\prime}\right)}{v}+\frac{g \beta *\left(C^{\prime}-C_{\infty}^{\prime}\right)}{v}=\frac{1}{v} \frac{\partial u}{\partial t^{\prime}} \\
\frac{\partial^{2} T^{\prime}}{\partial r^{2}}+\frac{1}{r} \frac{\partial T^{\prime}}{\partial r}=\frac{1}{\alpha} \frac{\partial T^{\prime}}{\partial t^{\prime}} \\
\frac{\partial C^{\prime}}{\partial t^{\prime}}=\frac{D}{r}\left[r \frac{\partial^{2} C^{\prime}}{\partial r^{2}}+\frac{\partial C^{\prime}}{\partial r}\right]-k_{l}\left(C^{\prime}-C_{\infty}^{\prime}\right)
\end{gathered}
$$

With boundary conditions 


$$
\left.\begin{array}{c}
t^{\prime} \leq 0, u=0, T^{\prime}=T_{\infty}^{\prime}, C^{\prime}=C_{\infty}^{\prime} \text { for all } r \\
t^{\prime}>0, u=u_{0}, \frac{\partial T^{\prime}}{\partial r}=-\frac{q_{w}}{k}, C^{\prime}=C_{w}^{\prime} \text { at } r=r_{0} \\
u \rightarrow 0, T^{\prime} \rightarrow T_{\infty}^{\prime}, C^{\prime} \rightarrow C_{\infty}^{\prime} \text { as } r \rightarrow \infty
\end{array}\right\}
$$

Introducing non-dimensional quantities

$$
\left.\begin{array}{c}
R=\frac{r}{r_{0}}, U=\frac{u}{u_{0}}, t=\frac{t^{\prime} v}{r_{0}^{2}}, T=\frac{T^{\prime}-T_{\infty}^{\prime}}{\frac{r_{0} q_{w}}{k}}, C=\frac{C^{\prime}-C_{\infty}^{\prime}}{C^{\prime}{ }_{w}-C_{\infty}^{\prime}}, \operatorname{Pr}=\frac{v}{\alpha}, S c=\frac{v}{D} \\
G r=\frac{g \beta q_{w} r_{0}^{2}}{v k u_{0}}, G c=\frac{g \beta^{*}\left(C_{w}^{\prime}-C_{\infty}^{\prime}\right) r_{0}{ }^{2}}{v u_{0}}, k_{i}=\frac{k_{t} r_{0}{ }^{2}}{v}
\end{array}\right\}
$$

The governing equations (1), (2) and (3) reduce to

$$
\begin{gathered}
\frac{\partial^{2} U}{\partial R^{2}}+\frac{1}{R} \frac{\partial U}{\partial R}+G r T+G c C=\frac{\partial U}{\partial t} \\
\frac{\partial^{2} T}{\partial R^{2}}+\frac{1}{R} \frac{\partial T}{\partial R}=\operatorname{Pr} \frac{\partial T}{\partial t} \\
\frac{\partial^{2} C}{\partial R^{2}}+\frac{1}{R} \frac{\partial C}{\partial R}=S c\left[\frac{\partial C}{\partial t}+k_{i} C\right]
\end{gathered}
$$

And corresponding initial and boundary conditions are

$$
\left.\begin{array}{c}
t \leq 0, U=0, T=0, C=0 \text { for all } R \\
t>0, U=1, \frac{\partial T}{\partial R}=-1, C=1 \text { at } R=1 \\
U \rightarrow 0, T \rightarrow 0, C \rightarrow 0 \text { as } R \rightarrow \infty
\end{array}\right\}
$$

\section{SOlution TechniQue}

We use the Laplace Transform technique to solve the above equations (6)-(8) subject to initial and boundary conditions (9). We obtain the temperature, concentration and the velocity profiles as follows:

$$
\begin{aligned}
& T=-\frac{2}{\pi} \int_{0}^{\infty}\left(1-e^{\left(-V^{2} t / \mathrm{Pr}\right)}\right) \Gamma_{3}(R, V) \frac{d V}{V^{2}} \\
& C=1+\frac{2}{\pi} \int_{0}^{\infty} e^{-\left(V^{2} / S c+k_{i}\right) t} \Gamma_{1}(R, V) \frac{V d V}{\left(V^{2}+k_{i} S c\right)} \\
& \text { For } \operatorname{Pr}=S c=1 \\
& U=1+\frac{2}{\pi} \int_{0}^{\infty} e^{-V^{2}} \Gamma_{1}(R, V) \frac{d V}{V}+\frac{G r}{\pi} \int_{0}^{\infty}\left(1-e^{-V^{2} t}\right)\left[R \Gamma_{2}(R, V)-\Gamma_{1}(R, V)\right] \frac{d V}{V^{3}} \\
& +\frac{2 G c}{\pi k_{i}} \int_{0}^{\infty} e^{-V^{2} t}\left[\Gamma_{1}\left(R, \sqrt{V^{2}+k_{i}}\right)-\Gamma_{1}(R, V)\right] \frac{V d V}{V^{2}+k_{i}} \\
& \text { For } \operatorname{Pr} \neq 1 \text { and } S c \neq 1 \\
& U=1+\frac{2}{\pi} \int_{0}^{\pi} e^{-r^{2}} \Gamma_{1}(R, V) \frac{d V}{V}+\frac{2 G r}{(\operatorname{Pr}-1) \pi} \int_{0}^{\pi}\left[V^{2} t+\operatorname{Pr}\left(e^{\left(r^{2} / / r_{1}\right)}-1\right)\right] \xi(R, V, \operatorname{Pr}) \frac{d V}{V^{4}} \\
& +\frac{2 G C S C}{\pi} \int_{0}^{\pi}\left(1-e^{-\left(V^{2} / S c+k_{i}\right)}\right)\left[\Gamma_{1}\left(R, \sqrt{V^{2} / S c+k_{i}}\right)-\Gamma_{1}(R, V)\right] \frac{V d V}{\left(V^{2}+k_{i} S C\right)\left(V^{2} S c-V^{2}-k_{i} S C\right)}
\end{aligned}
$$

Non-dimensional skin friction $\left.\tau=\frac{\partial U}{\partial R}\right]_{R=1}$ can be found from the equations (12) and (13) for $\operatorname{Pr}=S c=1$ and $\operatorname{Pr} \neq 1$ and $S c \neq 1$ respectively as $\tau=\frac{2}{\pi} \int_{0}^{\infty} e^{-r^{2} t} \Gamma_{4}(V) d V+\frac{G r}{\pi} \int_{0}^{\infty}\left(1-e^{-V^{2} t}\right)\left[\Gamma_{5}(V)-\Gamma_{4}(V)\right] \frac{d V}{V^{2}}+\frac{2 G c}{\pi k_{i}} \int_{0}^{\infty} e^{-V^{2} t}\left[\sqrt{\left(V^{2}+k_{i}\right)} \Gamma_{4}\left(\sqrt{V^{2}+k_{i}}\right)-V \Gamma_{4}(V)\right] \frac{V d V}{\left(V^{2}+k_{i}\right)}$

$$
\begin{aligned}
& \tau=\frac{2}{\pi} \int_{0}^{\infty} e^{-V^{2} t} \Gamma_{4}(V) d V+\frac{2 G r}{(\operatorname{Pr}-1) \sqrt{\operatorname{Pr}} \pi} \int_{0}^{\infty}\left[V^{2} t+\operatorname{Pr}\left(e^{\left(-V^{2} / / p r\right)}-1\right)\right] \Gamma_{6}(V) \frac{d V}{V^{3}} \\
& +\frac{2 G c S c}{\pi} \int_{0}^{\infty} e^{-\left(V^{2} / S c+k_{i}\right) t}\left[\sqrt{\left(V^{2} / S c+k_{i}\right)} \Gamma_{4}\left(V^{2} / S c+k_{i}\right)-\right. \\
& \left.V \Gamma_{4}(V)\right] \frac{V d V}{\left(V^{2}+k_{i} S c\right)\left(V^{2} S c-V^{2}-k_{i} S c\right)}
\end{aligned}
$$

Non-dimenfional sherwood number $\left.S_{h}=-\frac{\partial C}{\partial R}\right]_{R=1}$ can be obtained from the equation (11) as

$$
S_{h}=\frac{2}{\pi} \int_{0}^{\infty} e^{-\left(V^{2} / S c+k_{i}\right) t} \Gamma_{4}(V) \frac{V^{2} d V}{\left(V^{2}+k_{i} S c\right)}
$$

$$
\begin{gathered}
\text { Where } \\
\Gamma_{1}(R, V)=\frac{J_{0}(R V) Y_{0}(V)-Y_{0}(R V) J_{0}(V)}{J_{0}^{2}(V)+Y_{0}^{2}(V)}, \\
\Gamma_{2}(R, V)=\frac{J_{1}(R V) Y_{1}(V)-Y_{1}(R V) J_{1}(V)}{J_{1}^{2}(V)+Y_{1}^{2}(V)}, \\
\Gamma_{3}(R, V)=\frac{J_{0}(R V) Y_{1}(V)-Y_{0}(R V) J_{1}(V)}{J_{1}^{2}(V)+Y_{1}^{2}(V)} \\
\Gamma_{4}(V)=\frac{J_{1}(V) Y_{0}(V)-Y_{1}(V) J_{0}(V)}{J_{0}^{2}(V)+Y_{0}^{2}(V)}, \\
\Gamma_{5}(V)=\frac{\left\{J_{2}(V) Y_{1}(V)-Y_{2}(V) J_{1}(V)\right\}+\left\{J_{1}(V) Y_{0}(V)-Y_{1}(V) J_{0}(V)\right\}}{2\left[J_{1}^{2}(V)+Y_{1}^{2}(V)\right]}
\end{gathered}
$$$$
\Gamma_{4}(V)=\frac{J_{1}(V) Y_{0}(V)-Y_{1}(V) J_{0}(V)}{J_{0}^{2}(V)+Y_{0}^{2}(V)},
$$

$\Gamma_{6}(V)=-\left[\frac{\left\{J_{1}(V) J_{0}(V)+Y_{1}(V) Y_{0}(V)\right\}\left\{J_{1}(V / \sqrt{\operatorname{Pr}}) Y_{0}(V / \sqrt{\operatorname{Pr}})-Y_{1}(V / \sqrt{\operatorname{Pr}}) J_{0}(V / \sqrt{\operatorname{Pr}})\right.}{\left\{J_{1}^{2}(V)+Y_{1}^{2}(V)\right\}\left\{J_{0}^{2}(V / \sqrt{\operatorname{Pr}})+Y_{0}^{2}(V / \sqrt{\operatorname{Pr}})\right\}}\right]$

$+\frac{\frac{2}{\pi V}\left\{J_{1}(V / \sqrt{\operatorname{Pr}}) J_{0}(V / \sqrt{\operatorname{Pr}})+Y_{1}(V / \sqrt{\operatorname{Pr}}) Y_{0}(V / \sqrt{\operatorname{Pr}})\right\}}{\left\{J_{1}^{2}(V)+Y_{1}^{2}(V)\right\}\left\{J_{0}^{2}(V / \sqrt{\operatorname{Pr}})+Y_{0}^{2}(V / \sqrt{\operatorname{Pr}})\right\}}$

$\xi\left(R, V, P_{r}\right)=\Gamma_{3}(R, V)-$

$\left[\frac{\left\{J_{1}(V) J_{0}(V)+Y_{1}(V) Y_{0}(V)\right\}\left\{J_{0}(R V / \sqrt{\operatorname{Pr}}) Y_{0}(V / \sqrt{\operatorname{Pr}})-Y_{0}(R V / \sqrt{\operatorname{Pr}}) J_{0}(V / \sqrt{\operatorname{Pr}})\right\}}{\left\{J_{1}^{2}(V)+Y_{1}^{2}(V)\right\}\left\{J_{0}^{2}(V / \sqrt{\operatorname{Pr}})+Y_{0}^{2}(V / \sqrt{\operatorname{Pr}})\right\}}\right]$

$+\frac{\frac{2}{\pi V}\left\{J_{0}(R V / \sqrt{\operatorname{Pr}}) J_{0}(V / \sqrt{\operatorname{Pr}})+Y_{0}(R V / \sqrt{\operatorname{Pr}}) Y_{0}(V / \sqrt{\operatorname{Pr}})\right\}}{\left\{J_{1}^{2}(V)+Y_{1}^{2}(V)\right\}\left\{J_{0}^{2}(V / \sqrt{\operatorname{Pr}})+Y_{0}^{2}(V / \sqrt{\operatorname{Pr}})\right\}}$

\section{RESULTS AND DISCUSSIONS}

Velocity profiles for different values of chemical reaction $k_{i}$ are shown in fig. 1 . It is noted that the velocity increases with decreasing values of chemical reaction parameter $k_{i}$. The thermal Grashof number $G r$ signifies the relative effect of the buoyancy force to the hydrodynamic viscous force. The mass Grashof number Gc signifies the ratio of the species buoyancy force to the viscous hydrodynamic force. Fig. 2 depicts the velocity profiles at $P r=0.71, S c=0.6$ and $k_{i}=0.1$ for different values of time, which shows that velocity increases as time $t$ increases. In fig. 3 it is observed that the temperature decreases with increase the value of prandtl number and increases with the increase the values of time $t$. Concentration profiles for various chemical reaction parameter $k_{i}$ are shown in fig.4. The effect of the chemical reaction parameter is dominant concentration field. It is seen that the concentration increases with increasing values of chemical reaction parameter $k_{i}$. Skin friction for different values of chemical reaction parameter $k_{i}(=0.1,1.0,2.0), \operatorname{Pr}=0.71, S c=0.6 \quad$ and $\quad G r=G c=0.4 \quad$ are presented in fig.5.In this figure skin friction increases with increase in the values of $\mathrm{K}_{\mathrm{i}}$. Sherwood number for various parameters are shown in fig. 6.Sherwood number decreases with increasing values of chemical reaction parameter $k_{i}$. But it increases with increase in Schmidt number. 


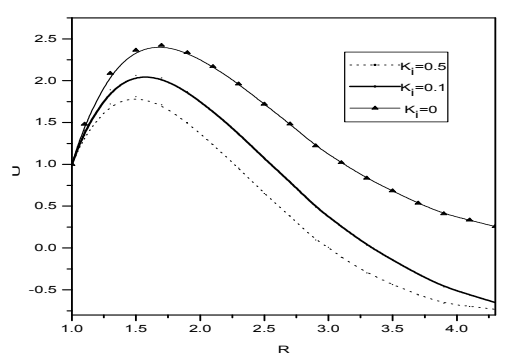

Fig. 1

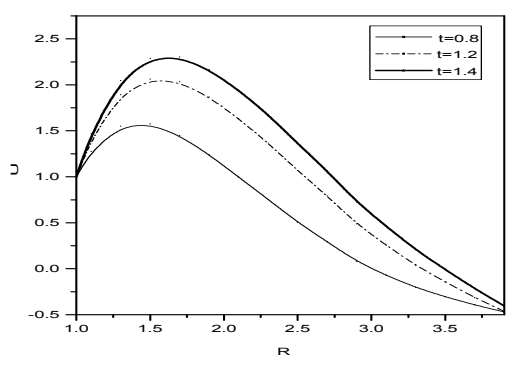

Fig. 2

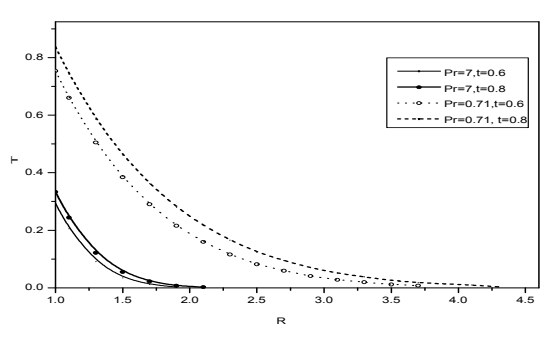

Fig. 3

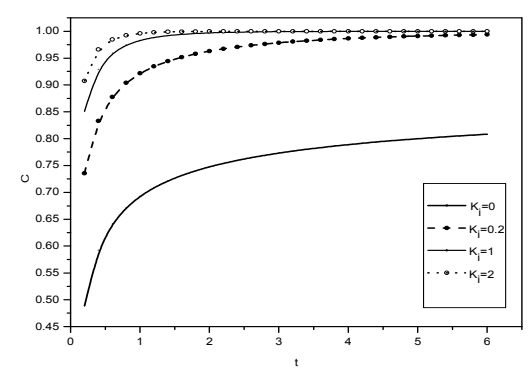

Fig. 4

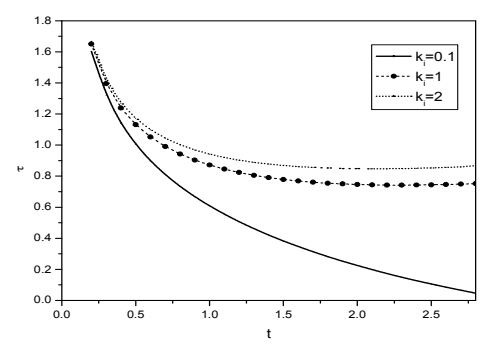

Fig. 5

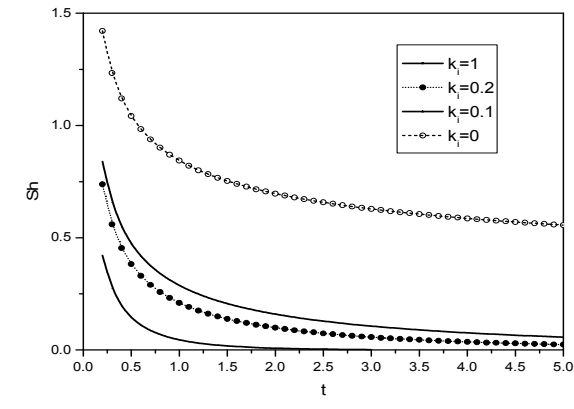

Fig. 6

\section{Conclusions}

Conclusions of this study are as follows:

1) It is observed that the velocity increases with increasing values of $G r$. But trend is reversed with respect to the Schmidt number, Prandtl number and chemical reaction parameter.

2) Velocity decreases with increasing the values of chemical reaction parameter, but opposite effect is observed for concentration profile.

3) Velocity and temperature profiles are decreasing as $\mathrm{Pr}$ increases.

4) In case of $S c$, the velocity and concentration profiles are decreasing as $S c$ increases.

5) Skin friction increases with decrease in $\mathrm{Pr}$ and increases with increase in $S c$ and $k_{i}$.

6) Sherwood number increases as Sc increases but it decreases as $k_{i}$ increases.

\section{REFERENCES}

[1] U. N. Das, R. K. Deka, and V. M. Soundalgekar, "Effects of mass transfer on flow past an impulsively started infinite vertical plate with constant heat flux and chemical reaction," Forschung im Ingenieurwesen, vol. 60, no. 10, pp. 284-290, 1994.

[2] P. Ganesan and P. Loganathan, "Heat and mass flux effects on a moving vertical cylinder with chemically reactive species diffusion," Journal of Engineering Physics and Thermophysics, vol. 75, pp. 899909, 2002.

[3] P. Ganesan and H. P. Rani, "On diffusion of chemically reactive species in convective flow along a vertical cylinder," Chemical Engineering and Processing, vol. 39, pp. 93-105, 2000.

[4] R. Muthucumaraswamy and M. Ravi Shankar, "First order chemical reaction and thermal radiation effects on unsteady flow past an accelerated isothermal infinite vertical plate. Indian," Journal of Science and Technology, vol. 4, pp. 573-577, 2004.

[5] P. Ganesan and P. Loganathan, "Radiation and mass transfer effects on flow of an incompressible viscous fluid past a moving vertical cylinder," International Journal of Heat and Mass Transfer 45, pp. 4281-4288, 2005.

[6] R. J. Goldstein and D. G. Briggs, "Transient free convection about a vertical plates and circular cylinders. Trans," ASME C. J. Heat transfer 86, pp. 490-500,1964. 